\title{
Zero-field optical manipulation of magnetic ions in semiconductors
}

R. C. MYERS, M. H. MIKKELSEN, J.-M. TANG, A. C. GOSSARD, M. E. FLATTÉ AND D. D. AWSCHALOM

Nature Materials 7, 203-208 (2008).

Owing to a printing error, the character denoting exchange interaction appeared incorrectly, it should have appeared as $\mathcal{J}$. The corrected text is below:

Page 3, second column, second paragraph:

For a Heisenberg hamiltonian $H=\sum_{i<i} \mathcal{J}\left(R_{i}-R_{j}\right) J_{i} \cdot J_{j}$, where $i$ and $j$ label all the Mn spins in the solid at position $R, \mathcal{J}=\sum \sum_{i} \mathcal{J}\left(R_{i}\right)$ and $\lambda=\mathcal{J}\left\langle J_{\mathrm{Mn}}\right\rangle$. Using methods developed previously ${ }^{17,19}$, we calculate the average interaction energy between two Mn spins at the average separation for a given density by using an effective Bohr radius of $13 \AA$. We estimate $\mathcal{J}$ from the interaction energy...

Page 5, second column, first line:

Assuming the values of $\mathcal{J}$ calculated above... 